\title{
Effect of intraoperative Ultrasonography on change of surgical decision for patients with hepatocellular carcinoma
}

\begin{abstract}
Background: Hepato cellular carcinoma (HCC) is one of the most common cancers worldwide. Intra operative ultrasonography (IOUS) is considered an indispensable operative procedure for intra operative decision-making and guidance of surgical procedures. IOUS had changed hepatic surgery dramatically because it was the only modality that was capable of delineating and examining the interior of the liver during surgery especially after incorporation of color Doppler imaging and laparoscopic ultrasound into IOUS in1990s. IOUS can accurately define the hepatic segmental boundaries as well as the tumor boundaries. The use of IOUS started in Japan and from there it spread worldwide allowing for the development of different therapies to treat patients with HCC. Our aim was to determine if IOUS can changes surgical decision in hepatic resection for $\mathrm{HCC}$.
\end{abstract}

Methods: We retrospectively analyzed 48 consecutive liver resections performed at multiple centers over 3years. Preoperative surgical decision was based on ultrasonography, computed tomography and/or dynamic magnetic resonance imaging (DMRI). The size, location and number of lesions were determined by IOUS and compared with preoperative imaging. Reviewing the operative report helped determine if new IOUS findings led to changes in surgical strategy. Pathology reports were analyzed for margins.

Results: This study was conducted on $48 \mathrm{HCC}$ consecutive patients 27 males and 21 females with age range from 49 to 67years. Hepatic resection was decided as the treatment of choice for all patients. During operation direct inspection and palpation of the liver followed by IOUS were done. The operative decision was changed in 17 patients $(35.4 \%)$, this was due to finding new additional tumors in 13 cases, and 4 cases showed change in tumour size on background of cirrhosis.

Conclusion: We found that IOUS findings directly changed the operative surgical decision in $35.4 \%$ of cases. Surgery was more accurately performed with IOUS. Despite the improvement of preoperative imaging modalities, IOUS can correctly define the hepatic segments as well as the extent of a tumor so it help for best operative decision in hepatic resections.

Keywords: hepato cellular carcinoma, hepatic surgery and intra operative ultrasonography, trans arterial chemo embolization
Volume I Issue 2 - 2016

\author{
Mohamed Mogahed,' Amr Abdelraouf,' \\ Ashraf Elkholy, 'Wessam M Abdellatif, ${ }^{2}$ Ashraf \\ Anas Zytoon ${ }^{3}$ \\ 'Surgical Department, National Hepatology and Tropical \\ Medicine Research Institute, Egypt \\ 2Radiology Department, National Hepatology and Tropical \\ Medicine Research Institute, Egypt \\ ${ }^{3}$ Radio diagnosis Department, Menoufiya University, Egypt
}

Correspondence: Mohamed Mogahed, National Hepatology and Tropical Medicine Research Institute, Surgical Department, Cairo, Egypt, Email mogahed 1968@yahoo.com

Received: November 02, 2016 | Published: November 29, 2016

\section{Introduction}

Hepato cellular carcinoma (HCC) is one of the most common cancers worldwide. Egypt has a high incidence of HCC (around $21 \%$ of cirrhotic Egyptian patients). This may be attributed to high prevalence of HCV in Egypt. ${ }^{1}$ Well-compensated cirrhotic patients with single lesions are good candidates for liver resection. Intra operative ultrasonography (IOUS) is considered an indispensable operative procedure for intra operative decision-making and guidance of surgical procedures. It had changed hepatic surgery dramatically because IOUS was the only modality that was capable of delineating and examining the interior of the liver during surgery ${ }^{2,3}$ especially after incorporation of color Doppler imaging and laparoscopic ultrasound into IOUS in 1990s. ${ }^{4}$ Patients with chronic hepatitis or cirrhosis demand a parenchyma-sparing liver surgery to minimize the risk of postoperative liver failure, which still represents an important source of mortality. ${ }^{5,6}$ The use of IOUS started in Japan and from there it spread worldwide allowing for the development of different therapies to treat patients with HCC. ${ }^{4}$ IOUS can accurately define the hepatic segmental boundaries as well as the tumor boundaries. ${ }^{7,8}$

\section{Patients and method}

A multicenter retrospective study was conducted on $48 \mathrm{HCC}$ consecutive patients 27 males and 21 females with age range from 49 to 67years. This was in the period between April 2013 and July 2016. Full history, clinical examination and laboratory investigations including alpha fetoprotein were done to all patients. Preoperative abdominal ultrasound, Triphasic CT and /or Dynamic MRI scan was performed for all patients IOUS was done for all patients after inspection and palpation of the liver intra-operatively. Dedicated intra-operative transducers used for intra-operative US of the liver. Typically, 5-MHz side-fire T-shaped linear- or curvilinear-array transducers are used (in our study we used T-shaped transducer BK medical, type 8816, 4.3-10 MHz) (Figure 1A-1C). For liver imaging, 
the transducer should comfortably fit against the palm of the hand and between the fingers to allow imaging of both the high dome and the right lateral segments of the liver. Transducers have color Doppler flow and pulsed Doppler imaging capabilities and provide good nearfield resolution. IOUS guidance has clear advantages over transabdominal imaging guidance procedures with regard to accessibility, especially in procedures involving deeply located lesions or lesions hidden by overlying structures. ${ }^{9}$

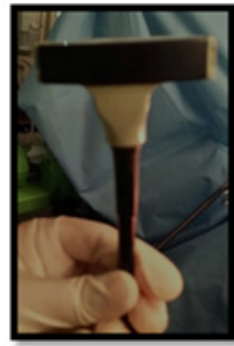

(1A)

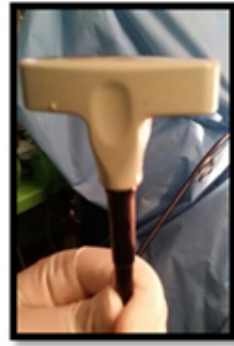

(1B)

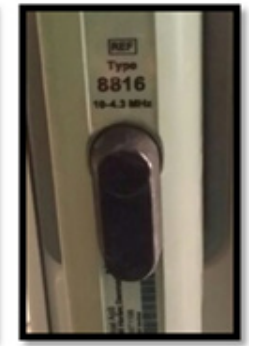

(1C)
Figure IA-IC T-shaped transducer, BK medical, type $8816,4.3-10 \mathrm{MHz}$.

The ultrasound machine should be placed in front of the first operator, with the aim to simultaneously view the screen and the operative field. The screen must be large enough to allow optimal visibility at that distance, and the assistants should then control the machine's keyboard that can be covered with a sterile drape. The liver exploration should always start with the inspection and palpation of the organ together with the entire peritoneal cavity.

Once in the peritoneal cavity, liver mobilization starts with the division of the falciform ligament as well as any adhesions to free the anterior-superior and inferior surfaces of the liver in order to get enough space to handle the probes for IOUS. Thus, by pulling the falciform ligament, the liver surface is widely exposed, and following the examination of the portal branches and hepatic veins, the entire liver can be studied. ${ }^{10}$ The planned operation which was open liver resection was documented in the last clinic note before operation. The actual procedure performed was recorded in the operative note. The IOUS was done and findings were reported by staff radiologist. The IOUS findings were compared with preoperative imaging reports. We recorded any differences in number, size or location of lesions and whether these findings led to a change in the operative plan. All resected HCC lesions were sent for histopathology.

\section{Statistical analysis}

Descriptive statistics were reported as frequencies and percents.

\section{Results}

Study was conducted on $48 \mathrm{HCC}$ consecutive patients 27 males and 21 females with age range from 49 to 67 years. Liver resection was decided for all patients. During operation direct inspection and palpation of the liver followed by IOUS were done. The operative decision was changed in 17 patients (35.4\%) this was due to the IOUS findings which were new additional tumors in 13 cases, and change in tumor size on background of cirrhosis in 4 cases as shown in Figure 1 \& Figure 2.

The 13 cases who had new lesions by IOUS the surgical decisions were changed as following:

A. 3 patients had the new lesions nearby the original tumor exten- ded resection was done. In 4 of the 17 cases the operations were aborted because significant increase in tumor size, vascular involvement, the liver morphology and residual parenchymal reserve would not be sufficient so they were referred for trans arterial chemo embolization (TACE) (Figure 3A-Figure 3F).

B. 10 patients had the new lesions away from the original tumor, 6 of them had resection of the original tumour together with intra operative radiofrequency while the remaining 4 had intra operative radiofrequency for both the original and new tumors. (Figure 3A- Figure 3D). The IOUS findings that led to changes in surgical plans are outlined in (Table 1) (Figure 4).

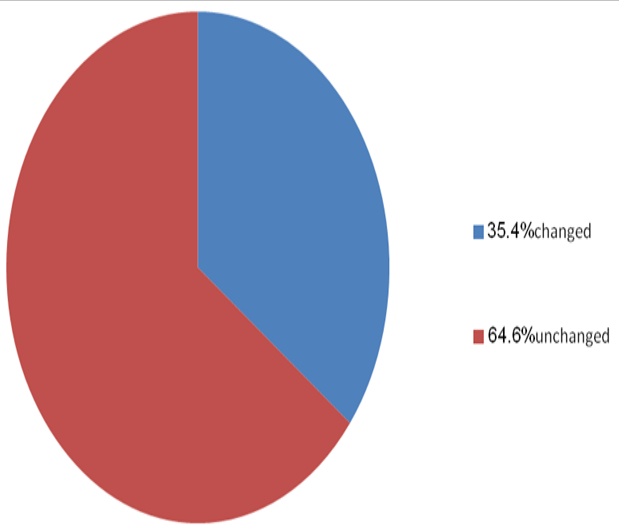

Figure 2 Number and \% of changed surgical plans after IOUS.

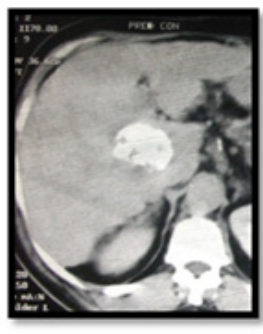

(3A)

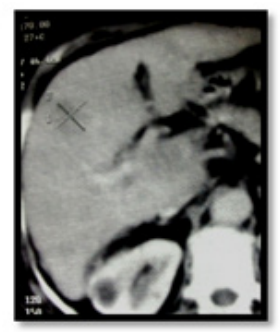

(3B)

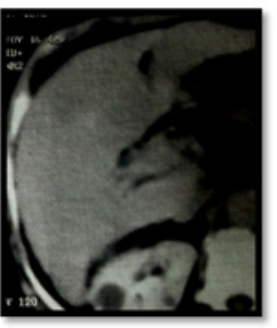

(3C)
Figure 3A-3C Triphasic CT images for a right hepatic lobe segment $\mathrm{V}$ lesion before doing IOUS.

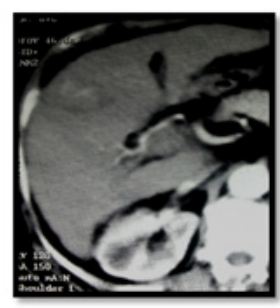

(3D)

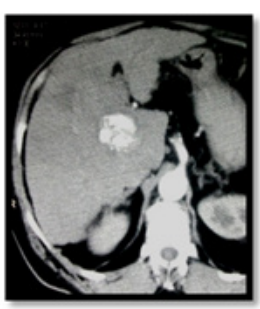

(3E)

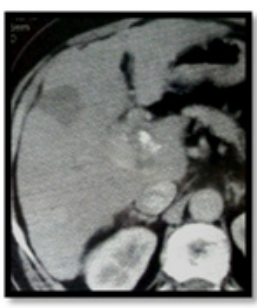

$(3 \mathrm{~F})$
Figure 3D-3F Triphasic CT for the same patient after doing IOUS with enlarged sized of the right lobe lesion and newly developed caudate lesion that treated by combined RFA and TACE.

Table I The IOUS findings that led to changes in surgical plans and number of cases

\begin{tabular}{ll}
\hline IOUS findings & Number and (\%) of cases \\
\hline Finding additional tumour & 13 cases $(27.1 \%)$ \\
Change in tumour size(marked increase) & 4 cases $(8.3 \%)$ \\
\hline
\end{tabular}




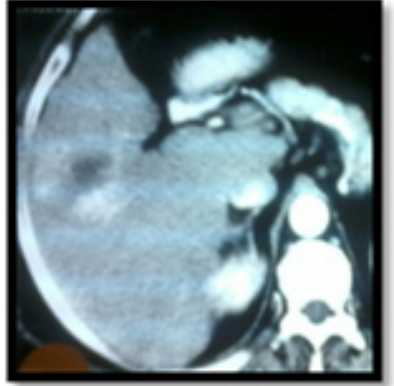

(4A)

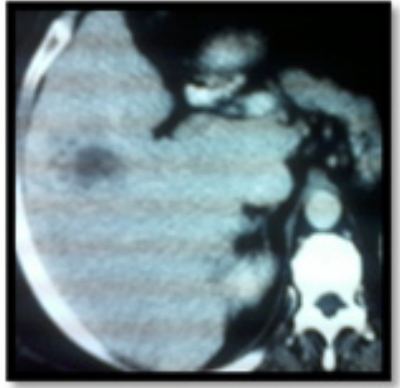

(4B)
Figure 4A \& 4B CT of right hepatic lobe segment $\mathrm{V} \mathrm{HCC}$ before doing intraoperative Ultrasonography.

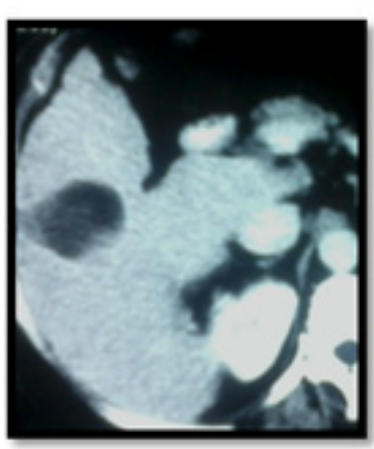

(4C)

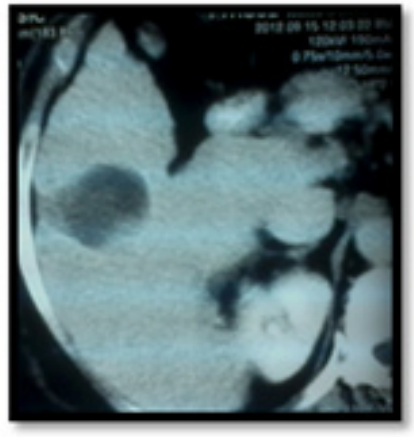

(4D)
Figure 4C \& 4D CT of the same lesion after RFA ablation (IOUS changes the decision due to close relation of the mass to the right portal vein.

\section{Discussion}

Hepatocellular carcinoma (HCC) is one of the most common cancers worldwide. Egypt has a high incidence of HCC in $21 \%$ of cirrhotic Egyptian patients. This may be attributed to high prevalence of Hepatitis C virus (HCV) in Egypt. ${ }^{1}$ The tumor number, tumor size, their location in the liver as well as the morphology of the underlying liver, together with other important parameters such as patient age, etiology, and residual parenchymal reserve, represent the main clinical parameters that are taken into consideration to favor one therapy over another. In particular, in patients indicated for surgery the preoperative staging should be very accurate to avoid useless and potentially risky operations.

Intraoperative ultrasonography (IOUS) is considered an indispensable operative procedure for intra operative decision-making and guidance of surgical procedures. It had changed hepatic surgery dramatically because IOUS was the only modality that was capable of delineating and examining the interior of the liver during surgery ${ }^{2,3}$ especially after incorporation of color Doppler imaging and into IOUS in $1990 \mathrm{~s} .{ }^{4}$ Our results showed that IOUS directly altered surgical plans in $35.4 \%$ of cases. Earlier studies published in the 1990s reported that surgical strategy was changed in up to $53 \%$ of cases., ${ }^{2,11-13}$ This can be explained by improvement in preoperative imaging modalities.

However Richy and his colleagues study on IOUS in 2015 reported $17 \%$ change in surgical strategy. ${ }^{14}$ This may be due to preoperative MRI were not done routinely to all our cases. In 4 of our 17 cases that changed due to IOUS findings of increased size of the tumor, the invasion of lesions to vascular structures played a major role in the operative decision, the operations were aborted and the 4 patients referred for TACE. 3 patients had the new lesions nearby the original tumor extended resection was done as the liver morphology and residual functional reserve fit for resection. patients had the new lesions away from the original tumor, resection of the original tumor plus intra operative radiofrequency for the new lesions were done. Patients had the new lesions away from the original tumor, intra operative radiofrequency for both original and new tumors were done as the location of the tumor, morphology of the liver, residual parenchymal reserve and patients' ages did not allow for resection.

\section{Conclusion}

We found that IOUS findings directly changed the operative surgical decision in $35.4 \%$ of cases. Surgery was more accurately performed with IOUS. Despite the improvement of preoperative imaging modalities, IOUS can correctly define the hepatic segments as well as the extent of a tumor so it helps for better operative decision in hepatic resections in patients with HCC on top of liver cirrhosis.

\section{Acknowledgements}

None.

\section{Conflict of interest}

Author declares that there is no conflict of interest.

\section{References}

1. Atti EA. HCC Burden in Egypt. Gastroentero J Hepatol Open Access. 2015;2(3):00045.

2. Luck AJ, Maddern GJ. Intraoperative abdominal ultrasonography. $\mathrm{Br} J$ Surg. 1999;86(1):5-16.

3. Ellsmere J, Kane R, Grinbaum R, et al. Intraoperative ultrasonography during planned liver resections: Why are we still performing it? Surg Endosc. 2007;21(8):1280-1283.

4. Makuuchi M, Torzilli G, Machi J. History of intraoperative ultrasonog raphy. Ultrasound Med Biol. 1998;24(9):1229-1242.

5. Torzilli G, Olivari N, Moroni E, et al. Contrast-enhanced intraoperative ultrasonography in surgery for hepatocellular carcinoma in cirrhosis. Liver Transpl. 2004;10(2Suppl 1):S34-S38.

6. Mark G Van Vledder, Timothy M Pawlik, Sanjay Munireddy, et al. Factors determining the sensitivity of intraoperative ultrasonography in detecting colorectal liver metastases in the modern era. Ann Surg Oncol. 2010;17(10):2756-2763.

7. Conlon R, Jacobs M, Dasgupta D. The value of intraoperative ultrasound during hepatic resection compared with improved preoperative magnetic resonance imaging. Eur J Ultrasound. 2003;16(3):211-216.

8. D Hondt M, Vandenbroucke Menu F, Préville Ratelle S, et al. Is intra-operative ultrasound still useful for the detection of a hepatic tumour in the era of modern pre-operative imaging? HPB (Oxford). 2011;13(9):665-669.

9. Jin Joo. The role of intraoperative ultrasonography in the diagnosis and management of focal hepatic lesions. Ultrasonography. 2015;34(4):246-257.

10. Matteo Donadon, Guido Torzilli. Intraoperative Ultrasound in Patients with Hepatocellular Carcinoma: From Daily Practice to Future Trends. Liver Cancer. 2013;2(1):16-24 
11. Parker GA, Lawrence W Jr, Neifeld JP, et al. Intraoperative ultrasound of the liver affects operative decision making. Ann Surg. 1989;209(5):569-576

12. Solomon MJ, Stephen MS, Gallinger S. Does intraoperative hepatic ultrasonography change surgical decision making during liver resection? Am J Surg. 1994;168(4):307-310.
13. Rifkin MD, Rosato FE, Branch HM, et al. Intraoperative ultrasound of the liver. An important adjunctive tool for decision making in the operating room. Ann Surg. 1987;205(5):466-472.

14. Ricky Jrearz, Richard Hart, Shiva Jayaraman. Intraoperative ultrasonography and surgical strategy in hepatic resection: What difference does it make? Can J Surg. 2015;58(5):318-322. 